\title{
Map Design for Public Health Emergencies: a Novel Conceptual Framework for Thematic Content Selection
}

\author{
Zixuan Han $^{1} \cdot$ Lingqi Wang $^{1}$ - Jiangyue Zhang ${ }^{1} \cdot$ Min Weng ${ }^{1} \cdot$ Mengjun Kang ${ }^{1}$
}

Accepted: 8 October 2021 / Published online: 29 October 2021

(c) The Author(s), under exclusive licence to Springer Nature Switzerland AG 2021

\begin{abstract}
Public health emergencies always lead to serious consequences which affect a lot on human health and socioeconomic progress. It is essential that governments and regional health commissions guide the public toward self-protection and better arranged social production during epidemic outbreaks and spreads. According to the need of risk communication and information disclosure, existing studies for COVID-19 maps and visualization applications are conducive to predicting the future trend of the pandemic, mitigating the harmful effect on public wellbeing by leading to effective intervention and policy measures. However, unsettled tasks remain on comprehensive organization of risk information, effective expression of data for public requirement, and systematic theoretical framework as a standard of map design for public health emergencies. To close the research gaps, this paper proposes a conceptual framework with a three-dimensional spatiotemporal-logic structure as a theoretical foundation for map thematic content selection, which is also a good basis for determining the effective visualization approaches of map design. It enhances the validity and legibility of the map expression by leading maps' thematic content couple with features and processes of an epidemic. Then, using the COVID-19 outbreak in Shenzhen, China, as an example, this paper illustrates how to apply the conceptual framework for selecting the thematic content of COVID-19 maps, and explains the specific ways to transform epidemic data into objects for cartographic representation with proper principles and modes. To our knowledge, this paper is the very first study to bring the thematic content of maps for public health emergencies to the fore, and it is thus believed to shed fresh lights into thematic map design.
\end{abstract}

Keywords Public health emergencies · COVID-19 map - Conceptual framework - Spatiotemporal-logic structure · Thematic content selection $\cdot$ Narrative map design

\section{Introduction}

Public health emergencies, both global and local, will always have serious consequences. These consequences affect not only human health, but also the economic progress of both developing and developed countries (Talisuna et al. 2020). As a major public health emergency that emerged at the end of 2019, COVID-19 posed a great threat to public safety, economic development, and peoples' personal wellbeing (Xiao 2020). It is essential that governments and regional health commissions guide the public toward self-protection as well as better coordinate and arrange social production

Mengjun Kang

mengjunk@whu.edu.cn

1 School of Resource and Environmental Sciences, Wuhan University, No.129 Luoyu Rd, Wuhan, Hubei Province, China during this pandemic using effective risk communication and information disclosure (Campbell-Lendrum et al. 2015; Zhang et al. 2020). Hence, epidemic outbreak numbers, generalized reports, web applications, in-depth investigations of official responses from authoritative media, and the mainstream internet media are all used as major forms of information exchange and disclosure that enable the public to understand the epidemic and its spatiotemporal risk pattern (Kim and Jung 2018; Xu et al. 2020a; Zhang L et al. 2020). To work out and delineate the pattern and relationships between human activities, epidemic evolution processes, and risk trends, many scientific research institutions, governmental institutions, and mainstream media outlets have thrown into COVID-19 scientific researches as well as design of different types of maps and geovisualization for this public health emergency (Akella 2009; Kostelnick and Hoeniges 2019; Ye et al. 2021). These maps and visualization forms integrated risk information to depict the dynamic patterns 
and properties of the epidemic as well as its adverse effect on the public from different aspects (Guillette et al 2020).

In essence, maps for public health emergencies belong to the category of emergency maps but are also quite different from traditional emergency maps. Emergency map is a typical form of expression for spatial geographic information related to major emergencies. Most of the traditional emergency maps are oriented to sudden natural disasters, of which the service objects typically are the government emergency management department and the relevant institutions. They are mostly applied to assist with scheduling and distribution of emergency resources, the generation of emergency plans, and the deployment of management work (Katzburg et al. 2020; Xu et al. 2020b). By comparison with a major emergency, a public health emergency has higher requirements for efficiency of information disclosure and cooperation between the government and the public due to its broader and acuter impacts on the public life. This leads to the discrepancies on service objects, data analysis, thematic content, and representation of the map design and compilation for public health emergencies (Brice 2020; Chapin and Sen Roy 2021), which is shown in Fig. 1. The main purpose of these adjustments on map design is to help the public holistically understand the spatiotemporal characteristics of the epidemic and implications of related policies from the limited and fragmented information available. Thus, these maps can implement features that reduce social panic and encourage the public to actively cooperate with the requirements and initiatives of the relevant departments and medical institutions that guard against the epidemic $(\mathrm{Xu}$ et al. 2020a).

In recent years, scholars have published many beneficial discussions and studies on emergency map design. Arco et al. (2017) constructed an implementation framework for emergency mapping services based on traffic signals. Vassileva et al. (2017) proposed an emergency mapping method based on radar interferometry. Elia et al. (2018) compared the effectiveness of using surveying and mapping data and crowdsourced data for emergency maps. Girres (2019) proposed an emergency mapping method for post-disaster loss
Fig. 1 Differences between traditional emergency map and maps for public health emergency




assessment based on remote sensing image comparison. Kostelnick and Hoeniges (2019) discussed the principle and method of emergency map symbol design in detail. Only a few studies have preliminarily discussed the theory and method of maps designed for public emergencies. Griffin et al. (2017) attempted to compare the portability of map design for different audiences. Kerkvoorde et al. (2018) evaluated the effectiveness of flood risk network map design on public understanding. Xu et al. (2020b) discussed the design of maps for earthquake emergencies based on audience theory. In brief, previous studies primarily focus on emergency map applications in disaster hazard analysis and are oriented to professionals or decision-makers.

There also have been some researches focused on observing dynamic patterns of COVID-19 by map design, visualizations, and application development. Yalcin (2020) presented cartograms of COVID-19 outbreak as a superior visualization tool to map the spread and spatiotemporal patterns of the epidemic. Guillette et al. (2020) conducted to use choropleth maps to visualize the geographic distribution of COVID-19 infection cases as an efficient tool to balance the need of risk disclosure with respecting individual privacy. Brice (2020) outlined a navigational approach for changing the information transmission forms of charts by converting time and space, aiming to optimize the maps' function of guiding the anticipation and interventions for emerging trend of COVID-19. Chapin and Sen Roy (2021) created a Web application of interactive visualizations at different spatial scales to reveal relationships between the patterns of COVID-19 and human mobility, helping users to understand areas affected by the pandemic and inform better suited policies for pandemic prevention. Liu F and Liu D (2020) proposed a visualization mapping analysis of emergency management scientific research big data. They used visual knowledge maps to summarize research trend of China's emergency management and point out the new ways to improve the response ability in the face of COVID19. Most of these studies are due to the requirements of governments and policy making for epidemic prediction and intervention, emphasizing on the overall trend and evolution feature analysis. They also concentrated on data analysis and visualization methods to inform one or a few aspects that may produce a specific impact on the epidemic dynamics, such as the spatial relationship between public risk of transmissions and human mobility (Chapin and Sen Roy 2021; Melin et al. 2020).

Although these policy-research-oriented maps and visualization applications have publicly released and covered a lot of information related to the public, and helped to mitigate the impact of the epidemic by coming up control measures, there are still some open questions. (1) Existing maps and visualizations are barely able to reveal the impact and the relationship of the epidemic on public life and their health circumstances directly from the public perspective. In addition, most data analysis and visualizations in larger geographic regions can easily drown the information heterogeneity between smaller ones and lead to misinterpretation of the epidemic risk and trend (Lovett et al. 2014). It is difficult for these data organization forms to directly help the public get the most relevant and useful information of their own living environment under the epidemics' threat and influence. (2) Few researches focused on the way to couple the visualization methods and forms with the epidemic evolution so that the public can intuitively extract the subject information addressed to their needs to encounter in the epidemic. Even though many studies have found that data visualization approaches are of great significance for informing and intervening in an epidemic, they still questioned their indicative functions to guide publics for epidemic prevention (Brice 2020). (3) There is a lack of systematic theoretical framework as a scientific foundation for integration, analysis, and expression of risk information for all the public health emergencies during a long time period. Researches of map design only taking COVID-19 as the core context may lead to limitations of results in application.

To close the research gaps, this paper constructs a conceptual framework for thematic content selection with predominant emphasis on comprehensive organization and expression of risk information at multi-spatiotemporal scales which couples with the narrative logic. As a universal theoretical methodology, this framework can be flexibly adjusted according to different target areas, event cycle, and trend. Then, using the COVID-19 outbreak in Shenzhen, China, as an example, this paper illustrates how to establish a threedimensional spatiotemporal-logic structure for selecting thematic content of COVID-19 maps. The Shenzhen case also explains the specific way and related visualization approaches to transform epidemic data to objects for cartographic representation with proper modes and principles. This study provides a guidance for the design of maps for public health emergencies, both in theory and in practice, aiming to depict the dynamics of the epidemic at different scales to reproduce and assess its evolutionary process and future trend as well as help the public understand their circumstances and roles in epidemic prevention.

\section{Conceptual Framework for Thematic Content Selection}

Focusing on key characteristics of a public health emergency should be the foundation of constructing a conceptual framework for map thematic content selection. Firstly, the occurrence of an unconventional major public health emergency such as COVID-19 is commonly with long cycle, wide influenced regions, and substantial 


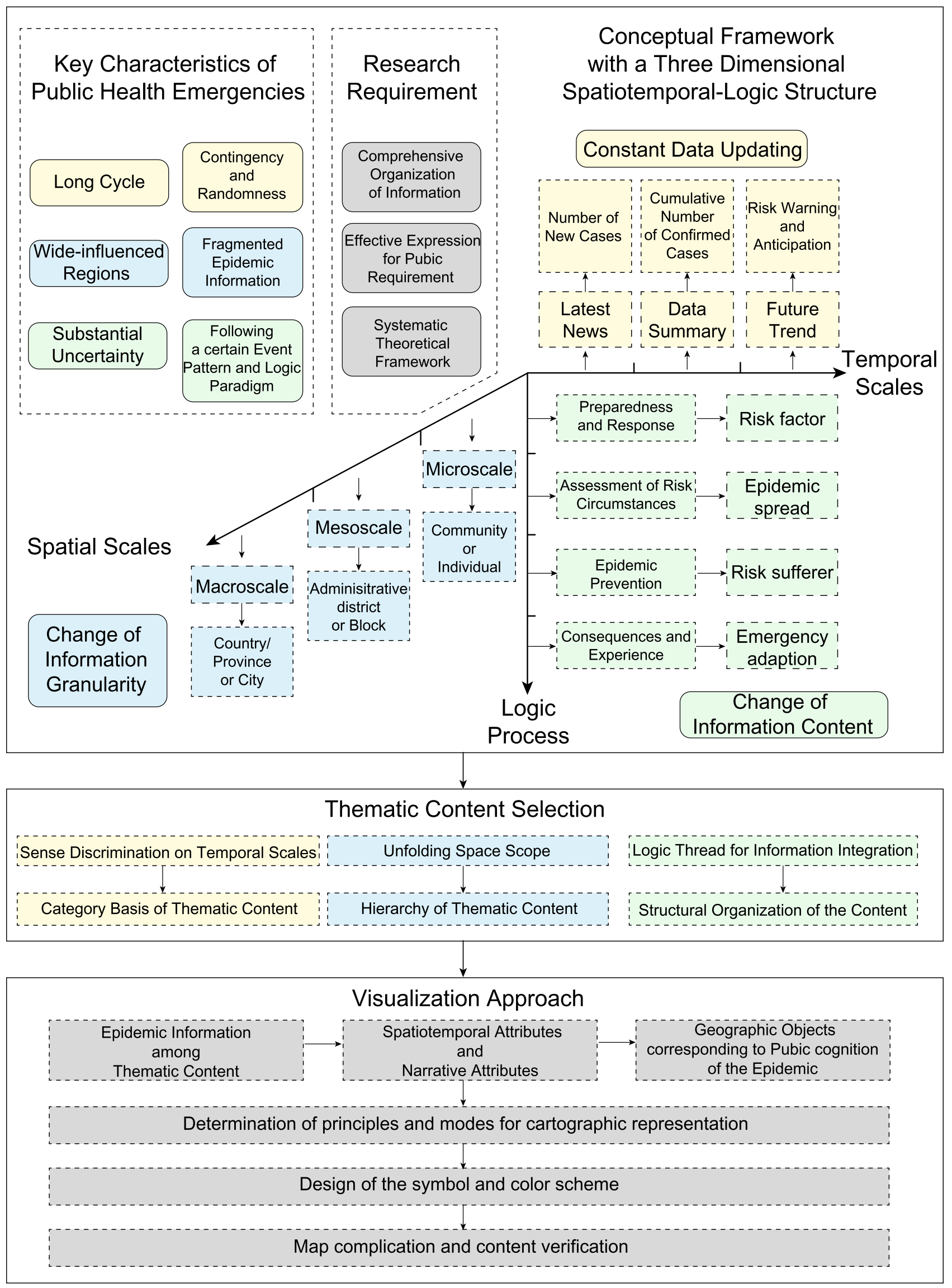


4Fig. 2 Conceptual framework for map thematic content selection

uncertainty. These events usually involve high contingency and randomness but also follow some certain dynamic patterns at complex spatiotemporal scales under a certain logical paradigm (Chapin and Sen Roy 2021). These elements indicate that relevant information regarding the epidemic is likely to be very fragmented and demands for continuous and comprehensive organization in the context of spatiotemporal changes as well as narratives among every event logic process (Pelizza 2020). Thus, forming and selecting the thematic content of maps for public health emergency need a framework with three-dimensional spatiotemporal-logic structure for information integration. Regarding spatiotemporal scales, marking time series of epidemic information due to data updating can help to sort out their order among event evolution while marking space changes can reflect the expansion of information granularity in spatial scales from coarse to fine. In addition, the logical connections between event processes are also needed as the content thread for integration and classification of information. This form of organization and expression can effectively balance the spatiotemporal attributes and narrative attributes of the epidemic information, better representing the evolutionary processes and dynamic patterns of a public health emergency (Rao et al. 2021).

On the one hand, the epidemic's temporal scale change leads to constant update of data in response to changes in the number of outbreaks and other quantitative indicators. Specifically, the latest news are expressed as the number of new cases while the data summary over a period of time represents the current epidemic circumstances with cumulative number of confirmed cases. Information in the context of epidemic prediction are more likely to be related with risk warning and prevention guidance. The sense discrimination on scales of time can be used as the category basis of maps' theme. On the other hand, spatial scale changes mean besides organizing the epidemic information at macroscales (such as the world, national, provincial, or city regions), mesoscales (such as administrative districts and blocks) and microscales (such as communities and individuals) are also needed (Zhou et al. 2020). The unfolding spatial scales also conduct to the hierarchy of thematic content. Besides, distinguishing these scales enables the maps to highlight heterogeneities of risk and epidemic's dynamics across different scopes of time and space. The more levels of spatiotemporal scale the map has, the more detailed the epidemic information is, and the public will therefore have access to more accurate information.

From the perspective of narrative logic, the response to a public health emergency corresponds to a four-step narrative logic process (Ario et al. 2019; Marome and Shaw 2021). First, preparedness and response to epidemic risk appear immediately after the occurrence of the emergency. The information demanded by the public during this process is primarily related to the outbreak numbers, namely, the risk factor. The second process is assessment of risk circumstances. This process involves gathering and communicating information about how the epidemic spreads, namely, the epidemic spread. The third process is epidemic prevention, which is closely related to the prevention and treatment of epidemic diseases. Thus, it is important to focus on the health of both individual patients and society as a whole, namely, the risk sufferer. Finally, the fourth process summarizes the consequences experienced during the emergency. In other words, the fourth process considers emergency adaption. This narrative logic is actually the content dimension of the epidemic information as well as a collection of semantic relations between spatiotemporal scales. The logic process presents characteristics of the epidemic and couples the information by the homogeneity of their attribute. In particular, as a kind of narrative map, the design of maps for public health emergencies emphasizes on information organization with narrative logic rather than the narrative cartographic representation.

The construction of the conceptual framework with a three-dimensional spatiotemporal-logic structure provides a theoretical foundation for map thematic content selection, which is also a good basis for determining the effective visualization approaches of map design. The spatiotemporal attributes and relations of the data give the map expression chances to pinpoint the epidemic information to specific situation and environment among publics' life. Besides, the data classification based on the logic process determines the way to transform the epidemic information into geographic objects according to their narrative attributes, which is essential to form visualization approaches for map design that is more corresponding to public cognitions. To a certain extent, this conceptual framework of map design for public health emergencies with the spatiotemporal-logic structure has changed the service mode of traditional maps. It enhances the validity and legibility of the map expression and is likely to gain more traction among the public and the government for more efficient cooperation confronting with the epidemic. Figure 2 shows the conceptual framework for map thematic content selection. 


\section{COVID-19 Map Design Case of Shenzhen, China}

\section{Study Area and Data Processing}

Shenzhen, located in the southern part of Guangdong Province, China $\left(113^{\circ} 43^{\prime} \sim 114^{\circ} 38^{\prime} \mathrm{E}, 22^{\circ} 24^{\prime} \sim 22^{\circ} 52^{\prime} \mathrm{N}\right)$, has nine administrative districts with a total area of $1997.47 \mathrm{~km}^{2}$ and a population of 17.56 million. It is a vice-provincial city and a super city as well as a special economic zone, a national economic center, and an international city of China. At the beginning of the COVID19 in 2020, the outbreak number in Shenzhen increased sharply due to the high density and massive mobility of the population. To help the public avoid potential risks and speed up the investigation of potential infection cases and close contacts, the relevant departments of the government have timely released the necessary epidemic data such as distributions of confirmed cases and their activity trajectory. Meanwhile, to improve the visualization of the published data, they also compiled and developed some thematic maps, electronic maps, and web applications for COVID-19. However, most of the maps still require the improvement in normalization, scientificity, and logicality of data organization and expression. Thus, this paper chose Shenzhen, China, as the study area for COVID19 map design, applying the conceptual framework for optimizing the thematic content selection and visualization approaches of the COVID-19 maps. Figure 3 shows the geographical location, administrative division, and scope of Shenzhen, which is available on the website of Shenzhen Platform for common Geospatial Information Services.

As an example of maps for public health emergencies, this case provides effective guidance for the similar map designs. According to the conceptual framework with the three-dimensional spatiotemporal-logic structure, Fig. 4 shows the overall flow chart for the map design of COVID19 in Shenzhen. The data requirements contained in our map design primarily include general statistics and the spatial distribution of the number of infection cases, close contacts, severe and critically ill patients, cases cured, and the deaths during the COVID-19 outbreak in Shenzhen as of 24:00 on February 3, 2020. All of the data used in this paper are either published online by relevant government departments and medical institutions or captured from the online map available through the open API interface.

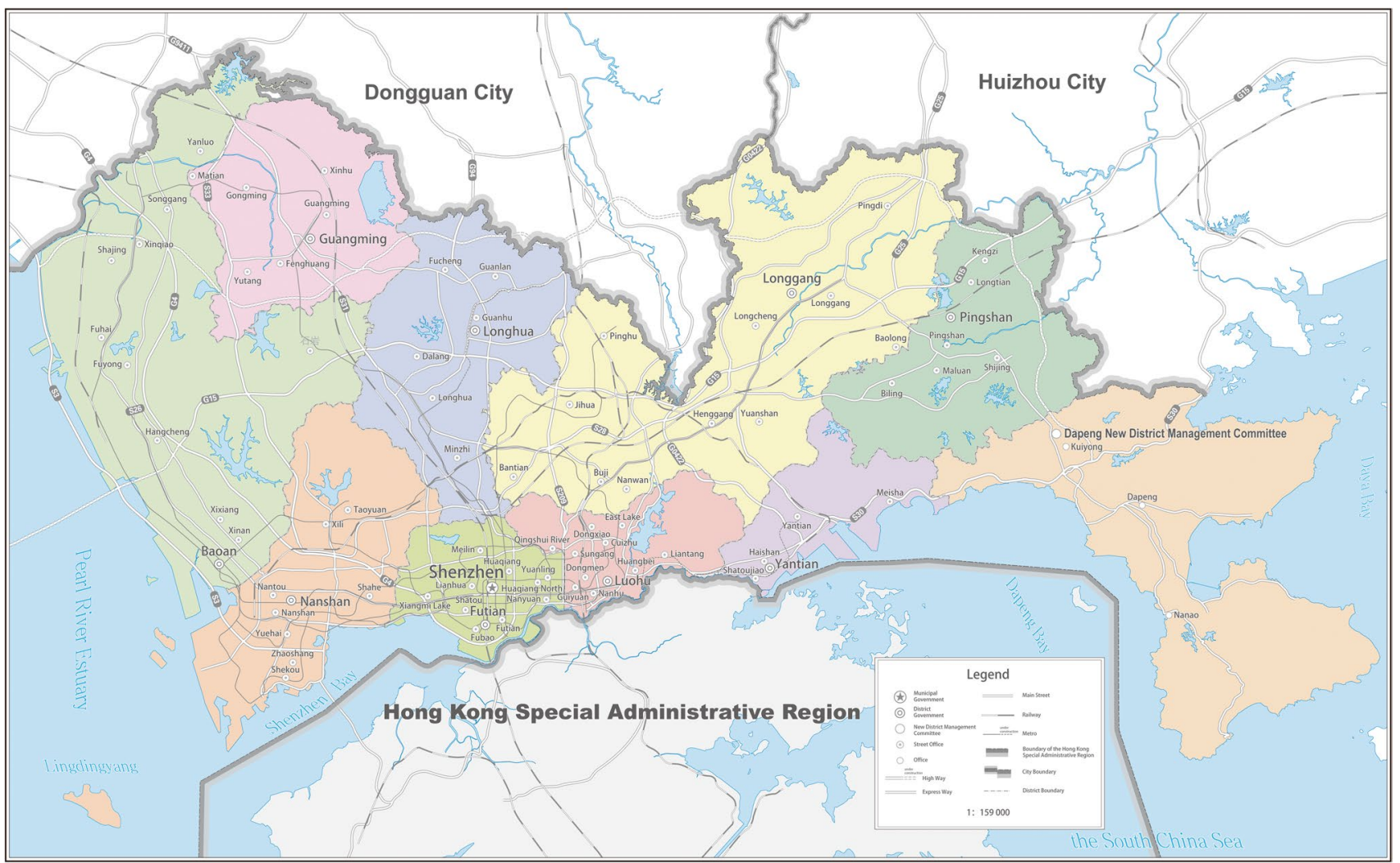

Fig. 3 Map of Shenzhen (https://guangdong.tianditu.gov.cn/shenzhen/bzmap) 
Fig. 4 Overall flow chart for map design of COVID-19 in Shenzhen

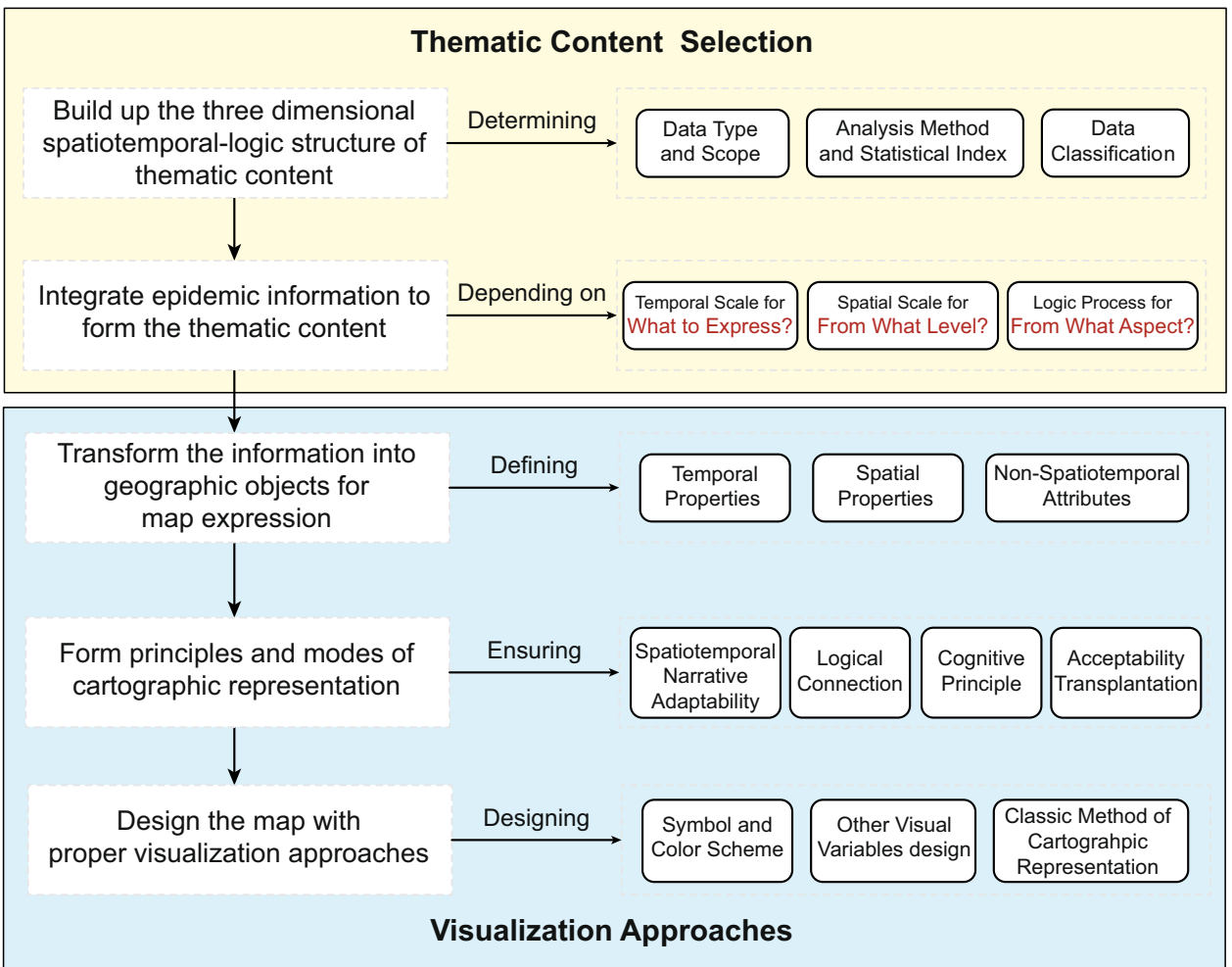

\section{Thematic Content Determination}

The conceptual framework with a three-dimensional spatiotemporal-logic structure proposes the demands of data gathering and organizing. Temporal scales can be set up according to time series of data generating and updating and their statistical scopes. Different temporal scales directly addressed to different information requirements of the public during the epidemic, were namely the latest dynamics delivery, epidemic circumstances summary, and future trend prediction. This determines the main theme of each COVID-19 map. To fully cover the space scopes that the epidemic impacted on the public life, setting of spatial scales should involve recognized basic unit of urban administration and also the focal place for the epidemic outbreak, spread, and prevention such as country, city, and community (Yang et al. 2021). Due to the features of wide urban area and large population density in Shenzhen, the map design

Table 1 Data classification depending on the narrative logic process

\begin{tabular}{|c|c|c|c|}
\hline Narrative logic process & Data category & Explanation & Data form \\
\hline Preparedness and response & Risk factor & $\begin{array}{l}\text { Information containing potential risk of } \\
\text { transmission }\end{array}$ & $\begin{array}{l}\text { Location, scope of activities of infection } \\
\text { cases, etc }\end{array}$ \\
\hline Assessment of risk circumstances & Epidemic spread & $\begin{array}{l}\text { A series of risk factors within a certain } \\
\text { range and route of transmission }\end{array}$ & $\begin{array}{l}\text { Relation among infection cases, aggrega- } \\
\text { tion and migration of the population, etc }\end{array}$ \\
\hline Epidemic prevention & Risk sufferer & $\begin{array}{l}\text { Groups or individuals affected and suf- } \\
\text { fered in the epidemic }\end{array}$ & $\begin{array}{l}\text { Peoples' personal wellbeing level } \\
\text { People's daily life order }\end{array}$ \\
\hline \multirow[t]{2}{*}{ Consequences and experience } & Emergency adaption & Negative impacts on the public & $\begin{array}{l}\text { The critical illness rate, mortality rate, } \\
\text { occurrence of aggregation cases } \\
\text { Public safety and social economic devel- } \\
\text { opment that have been damaged }\end{array}$ \\
\hline & & Positive work in epidemic prevention & $\begin{array}{l}\text { Cure rate } \\
\text { Efficient measures and contributions of } \\
\text { individuals, medical institutions and } \\
\text { relevant government departments for } \\
\text { epidemic prevention }\end{array}$ \\
\hline
\end{tabular}


SPATIAL

TEMPORAL

SCALE

SCALE
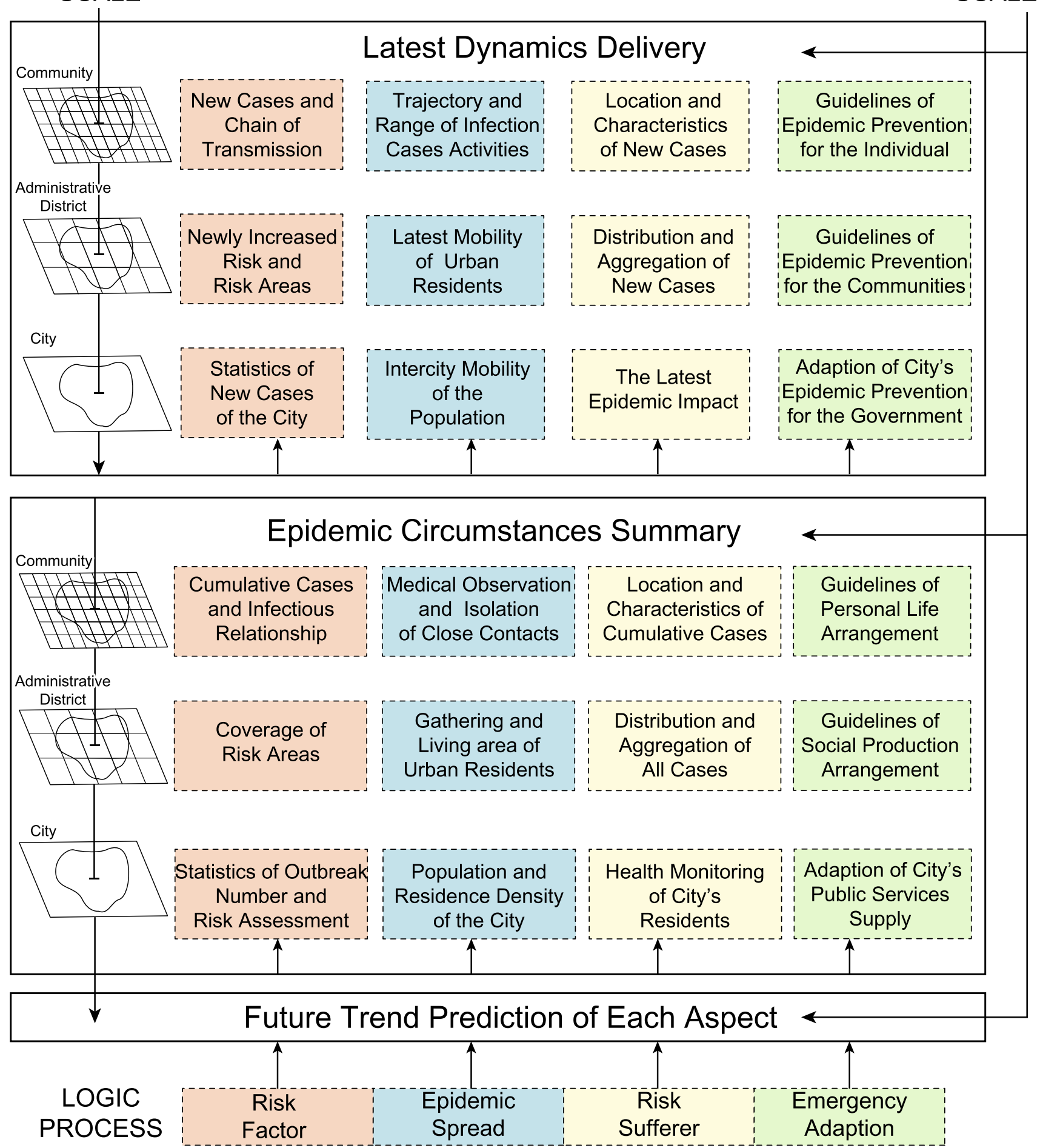

Fig. 5 Thematic content system of COVID-19 maps

of COVID-19 set a three-level spatial scale system at city, administrative district, and community scales to appear the macro tendency and spatial heterogeneity of the epidemic within the urban area respectively. Statistical indexes and analysis methods fitting with different spatial scales help to process and express the data from microscale to macroscale, 
Table 2 Specific visualization approach of the COVID-19 maps

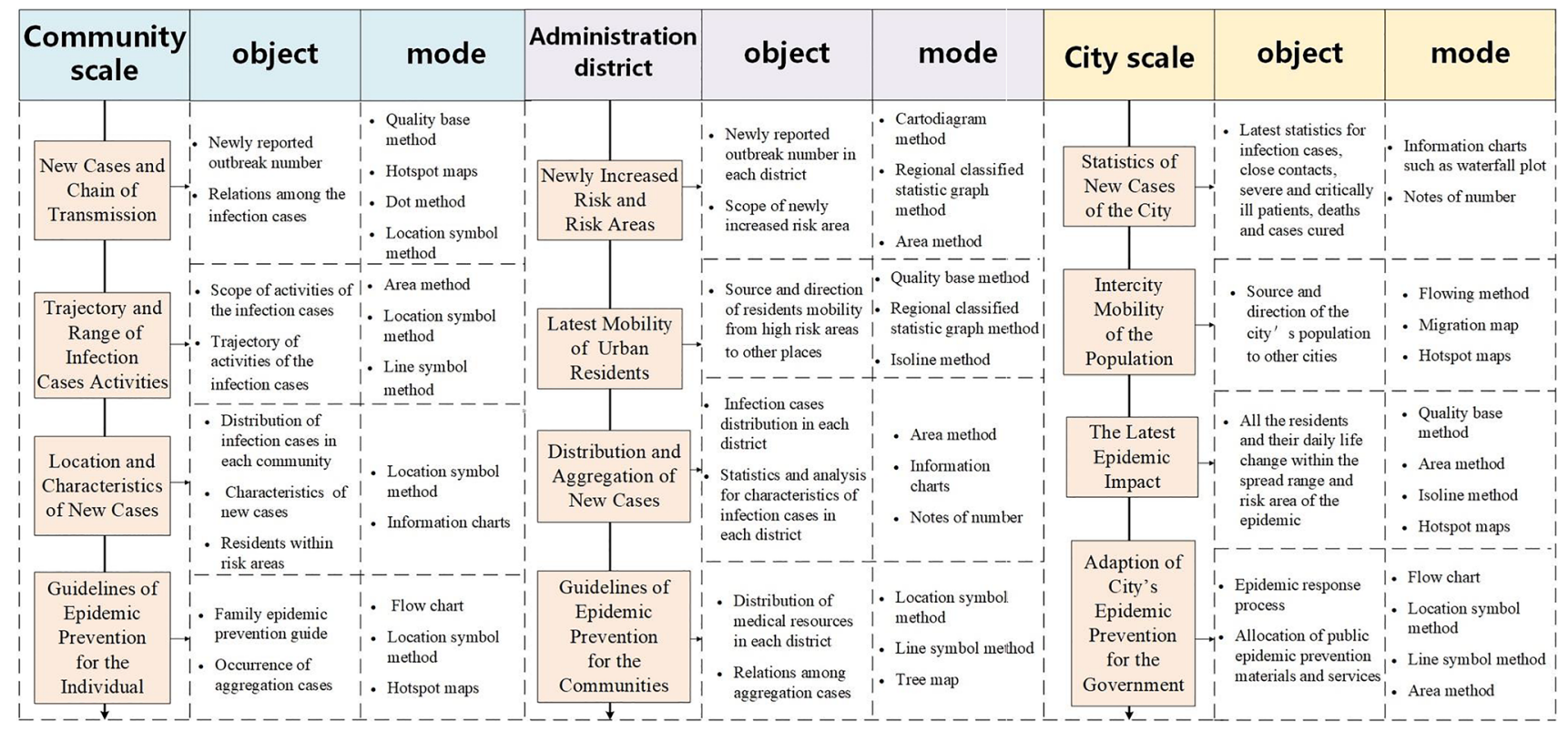

which determines the hierarchical scheme of maps' thematic content. Finally, to integrate data at each spatiotemporal scale into narrative structure, it is essential to assess which logic process of the epidemic data is related to and classify the data into four categories, according to their nonspatiotemporal attributes. Table 1 shows the method for the data classification.

As the connection of semantics of data at different spatiotemporal scales, the logic process couples the content and organization form of data presented on the maps with the features of epidemic evolution, which determines four narrative aspects of the thematic content. The final formed thematic content covers all logic processes at each spatiotemporal scale. Along with the spatiotemporal scales of data in four logical categories changing from micro to macro, the thematic content changes from concrete to general. Figure 5 shows the final thematic content system.

\section{Visualization Approach}

\section{Principles, Objects, and Modes of Cartographic Representation}

The visualization approaches determine the principles of map expression as well as the objects and proper modes of cartographic representation. To transmit the thematic content to map expression, the epidemic data needs to transform to geographic objects. This requires to define the spatiotemporal properties and attributes for the data according to their corresponding thematic content and their own characteristics. To clearly depict the specific geographic objects and their characteristics on the COVID-19 maps, it is also important to set appropriate principles and modes for cartographic representation depending on maps' thematic content (Wildbur and Burke 1998; Li 2021).

First, the cartographic representation should follow the basic requirements of specialization, standardization, and readability, ensuring the map expression is fully coincident with the thematic content. According to demands of COVID-19 map design based on the conceptual framework with the spatiotemporal-logic structure, the principles for determining the objects and modes of cartographic representation should be followed as:

(1) Principle of spatiotemporal narrative adaptability

The thematic content displays many differences at each spatiotemporal scale and narrative aspect. Setting appropriate schemes of color, symbol, and layout helps to characterize the objects corresponding to the thematic content of different levels (Weng et al. 2020).

(2) Principle of the logical connection

Clearly depicting the event logic on the map using the appropriate cartographic representation is the most effective way to guide readers toward understanding the dynamic changes and risk trends involved in the COVID-19 epidemic. Therefore, one important aspect of appropriate visualization is to create a sense of order using an appropriate symbol and color schemes.

(3) Second-order cognitive principle

This principle refers to providing useful, usable, understandable, and meaningful functional information from the perspective of readers' needs (Malpass 2016). This requires the design of symbol systems and color systems that represent the most salient cartographic 
Latest Dynamic Delivery at City \& Administrative District Scales



Fig. 6 Number of new cases in each district of Shenzhen

objects vividly, directly, and clearly. These objects often transform second-order information, processed by cartographers, from mathematical statistics, spatial analysis, and other technologies.

(4) Principle of acceptability transplantation

The theory of mass communication indicates that all readers utilize a pre-determined reference system to receive information (Malpass 2016). Using color design as an example, the use of red often indicates an increase in the outbreak numbers and therefore plays a role in warning the public (Adams et al. 2008; Landa 1996). Green, on the other hand, often indicates the number of people cured, bringing hope and comfort to the public (Adams et al. 2008; Landa 1996). This means that the COVID-19 maps need to adhere to this familiar and expected visualization. In particular, due to the high similarity of data forms, the representation modes in different temporal scales have a lot in common as well. Thus, combining both the principles and practical application, and based on the classical representation of thematic maps, the specific objects and modes of cartographic representation for the maps about Latest Dynamic Delivery are set and shown in Table 2.

\section{Examples of map design}

According to the thematic content system in Fig. 5, this paper presents 6 examples of map design, grouped by themes of Latest Dynamic Delivery, Epidemic Circumstances Summary and Future Trend and Prediction. These maps show the application effect of the conceptual framework of thematic content selection with the spatiotemporal-logic structure in practice. The maps mainly take the warm color scheme (yellow/red) complemented by cold color scheme (blue/gray) to indicate the epidemic risk and dynamics, which have been proved with remarkable strengths in visual cognition and understanding of the public (Fang et al. 2021). 
Latest Dynamic Delivery at Administrictive District \& Community Scales



Fig. 7 New cases in communities of Guangming and Longhua Districts of Shenzhen

(1) Latest dynamic delivery maps of new cases of COVID19 in Shenzhen

The COVID-19 map shown in Fig. 6 depicts the latest dynamic of COVID-19 of Shenzhen on the day of February 3,2020 , at city and administrative district scales. It utilizes choropleth map with highlight figure notes to show the number of new cases in each district of Shenzhen and their epidemic risk levels. In particular, cases directly sent to designated hospitals from transportation hubs, such as airports, stations, docks, and checkpoints, are expressed and explained in a separate graph because they are not counted as cases in any administrative district. The bar charts on polar with arrow symbols are used to reflect whether the new cases are imported or indigenous, indicating the direction of human mobility. Waterfall plots in the map display the daily update of COVID-19 outbreak numbers since the start of the epidemic, reflecting the absolute and relative changes in infection cases over time.
The map shows that Futian, Longhua, and Luohu districts have the largest number of new cases. It also reveals that the number of imported cases was significantly more than that of the indigenous cases in the early stage of the epidemic. In terms of the time series of case changes, the number of new cases continues to swell every day, indicating there are still great potential risks of epidemic aggravating.

COVID-19 map shown in Fig. 7 is about distribution and features of new cases at administrative district and community scales. It uses the quality base method and location symbol method to display the location and distribution of communities in which new cases were found and their surrounding residential areas, taking Guangming and Longhua districts as examples. The doughnut charts with figure notes demonstrate the number of new cases in each district and its proportion in the total number of new cases in Shenzhen as well as the population in each district and its proportion in city's population. The pictorial bars employed graphic elements of male and female depict the characteristics of new cases by statistic of their age and gender. The map shows 
Epidemic Circumstances Summary at City \& Administrative District Scales

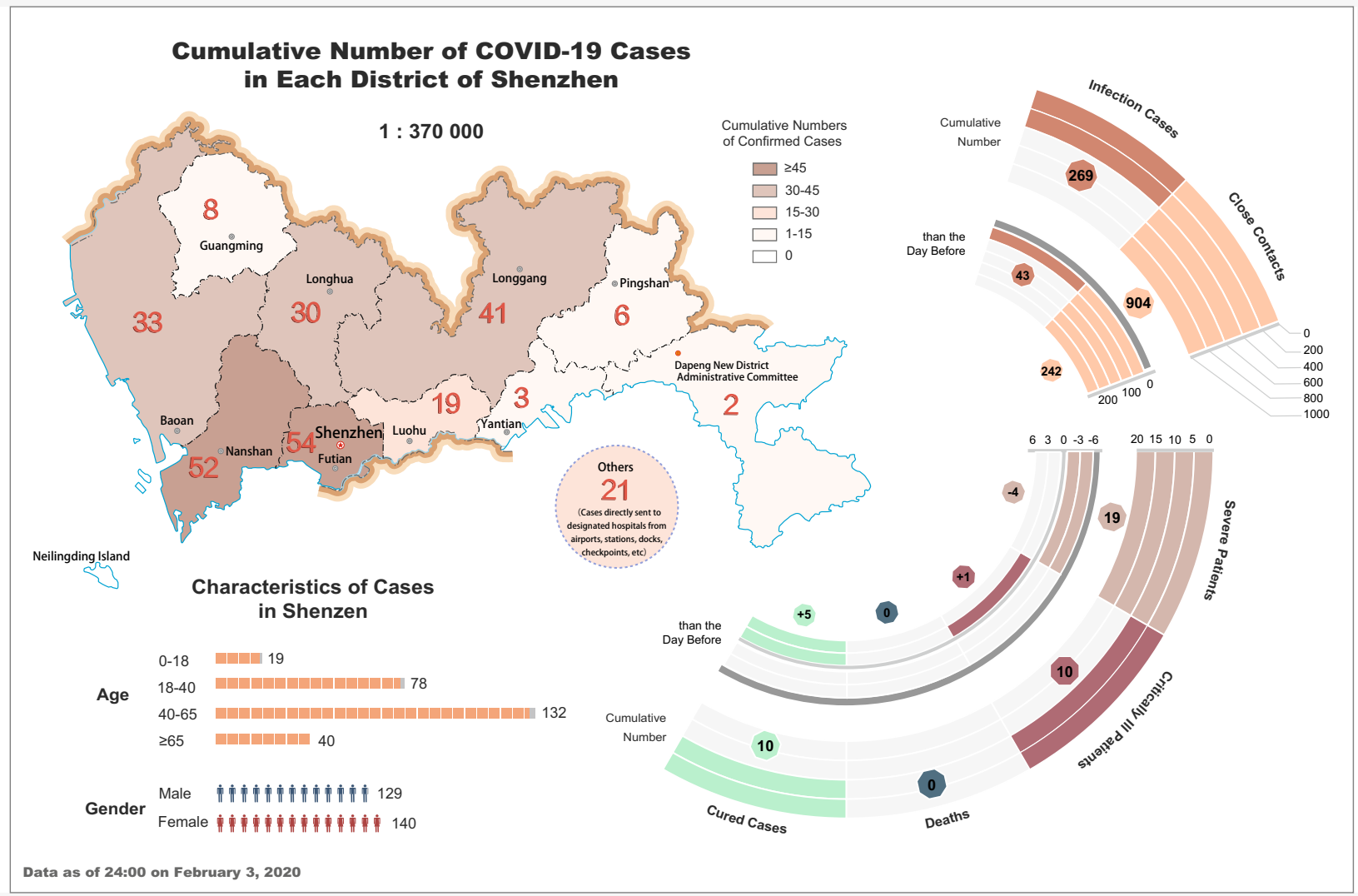

Fig. 8 General statistics for the COVID-19 outbreak in Shenzhen

the epidemic impact in Guangming and Longhua districts respectively and also directly present the spatial relation between communities with new cases and surrounding residential areas. It helps remind the residents nearby to pay close attention to their own health circumstances and reduce the arrangement of travelling and gathering.

(2) Epidemic circumstances summary maps of cumulative COVID-19 cases in Shenzhen

The COVID-19 map in Fig. 8 depicts the epidemic circumstances of COVID-19 in Shenzhen to date as of February 3, 2020, at city and administrative district scales, taking the choropleth map as the effective method to indicate the epidemic situation and risks. Two groups of bar charts on polar demonstrate the general statistics of infection cases, close contact, severe patients, critically patients, cured cases, and the deaths. The pictorial bar shows that the confirmed cases were mixed between men and women, but a large part of them were over 40 years old. This map plays an important role for the public to understand the epidemic pattern during its rapid spread.

Then, the map in Fig. 9 explains relationships among the typical COVID-19 aggregated cases mainly occurring in communities and families. As a tree map, it provides a detailed analysis of the beginning as well as the relation and time series of the multigenerational aggregated cases.

\section{(3) Future trend and prediction}

The COVID-19 map shown in Fig. 10 is about epidemic risk prediction of COVID-19 in Shenzhen. The combination of location symbol method, quality base method, and area method can demonstrate the possible risk areas through distributions of the infection cases and their possible walking range within $30 \mathrm{~min}$. The heat map with kernel density estimating can also present the spatial pattern of epidemic risk, leading the public to comprehensively grasp the epidemic situation and future risk trend. 
Epidemic Circumstances Summary of Community Scales

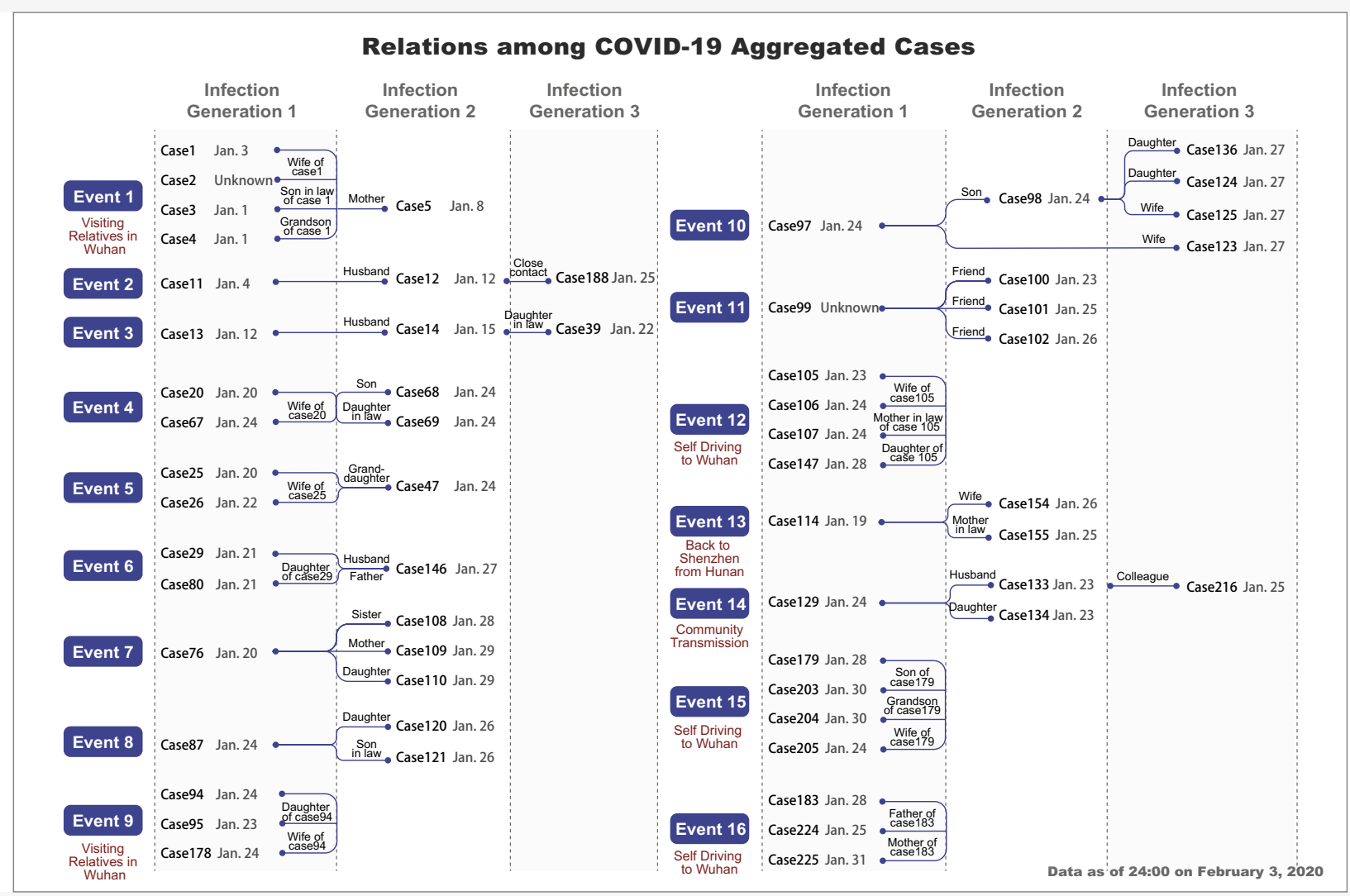

Fig. 9 Relations among the COVID-19 aggregated cases

The last one in Fig. 11 depicts the epidemic risk prediction of Guangming and Longhua districts. It also takes quality base method and area method to illustrate possible walking ranges of the cases as well as the residential areas that may be contained in the scope of high-level and medium-level risk areas. The assist information charts with highlight figure notes display the number of confirmed cases and the number of communities where they are found in each district. It is easy to notice that there are more infection cases along with higher density of residential areas and larger epidemic risk in Longhua district. The waterfall plots with infection cases that keep climbing indicate the inflection point of the epidemic in each district has not appeared yet. The map specially warns the residents living in the risk area and reminds the public about continued epidemic prevention and control efforts by revealing the future trend of the epidemic spread and evolution.

\section{Conclusions}

In view of the limitations of the research on map design for public health emergencies, this paper establishes a conceptual framework for thematic content selection with a threedimensional spatiotemporal-logic structure. As a theoretical foundation for determining the thematic content and visualization approaches of map design for health public emergencies, the framework conducts to depict the dynamics of the epidemic at different scales to reproduce and assess its evolutionary process and future trend, guiding the public to understand their circumstances and roles in epidemic prevention. It also uses the COVID-19 outbreak in Shenzhen as an example to illustrate the specific way and processes to provide theoretical and technical support for the map design. To our knowledge, this paper is the very first study to bring the thematic content of maps for public health emergencies to the fore. It has changed the service mode of traditional maps to a certain extent, enhancing and optimizing the 


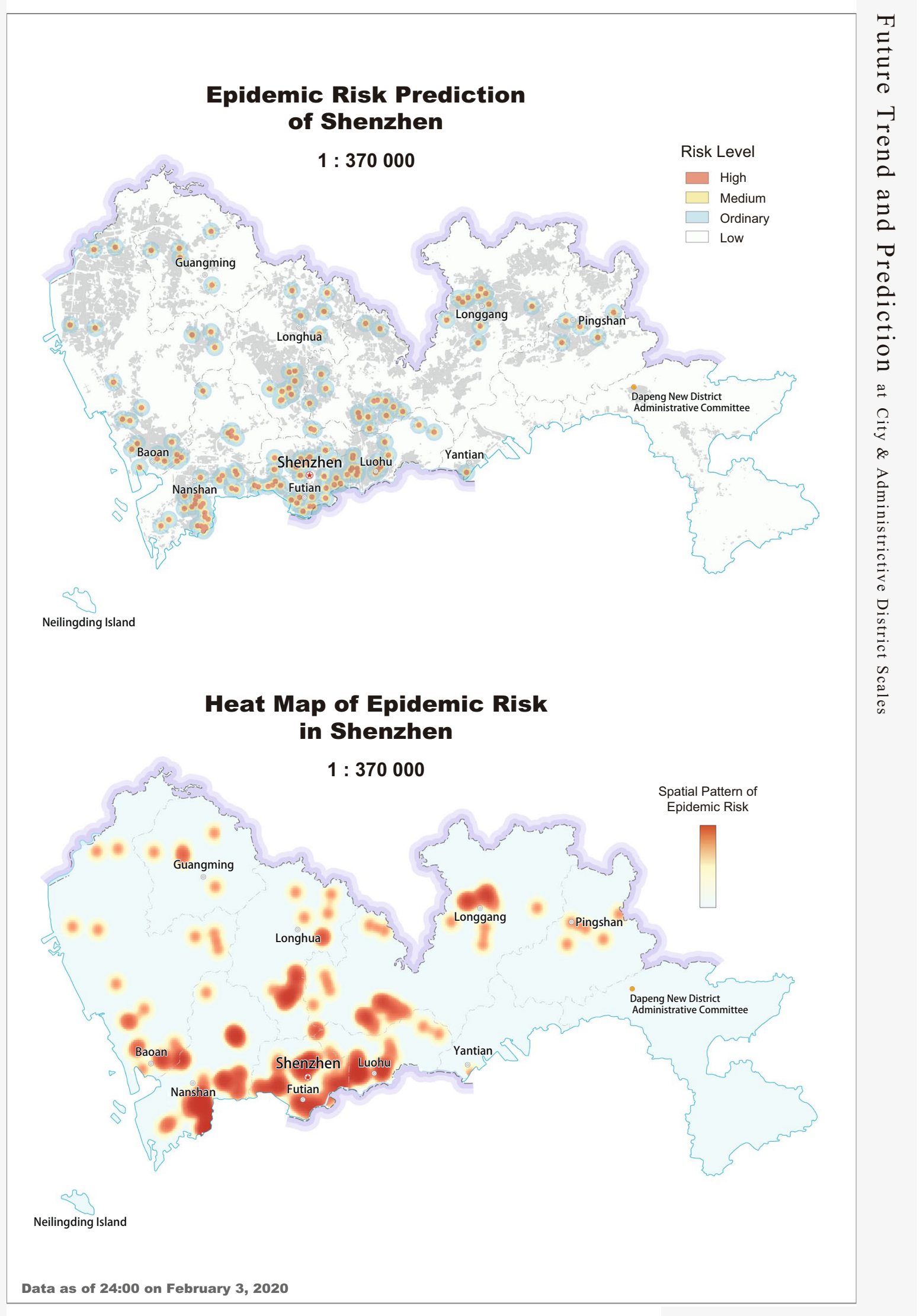


४Fig. 10 Epidemic risk spatial pattern and prediction of Shenzhen

rationality, validity, and legibility of map design for public health emergencies.

However, there are still some deficiencies in this study. First, due to the availability of epidemic information and related information on the COVID-19 outbreak, this paper does not provide maps that depict medical resource allocation or the arrangement of social production. Second, in the early stage of public health emergencies, there are likely to be gaps between data collection and data publishing. This problem can lead to deviations between the outcome maps and the currently available information, which was not discussed in this paper. Third, map samples have not carried out psychological or eye movement monitoring experiments as well, so there is still a lack of quantitative detection about the effectiveness of these theoretical and technical map design techniques. Besides, the study on theoretical framework and practice examples in this stage has not considered the accommodation of rapid information updates in the internet publishing environment and real-world applications yet. These limitations will certainly be explored in depth in related research. In addition, developing a semi-automatic cartographic system depending on the theoretical framework for more efficient production of the maps and designing a digital global-style geovisualization platform for more stereoscopic and comprehensive map expression would also be worth exploring in our future study.

\section{Software}

ESRI ArcGIS 10.2 was used for statistical analysis. Adobe Illustrator CC 2019 was used for map design.

Future Trend and Prediction at Administrative District \& Community Scales

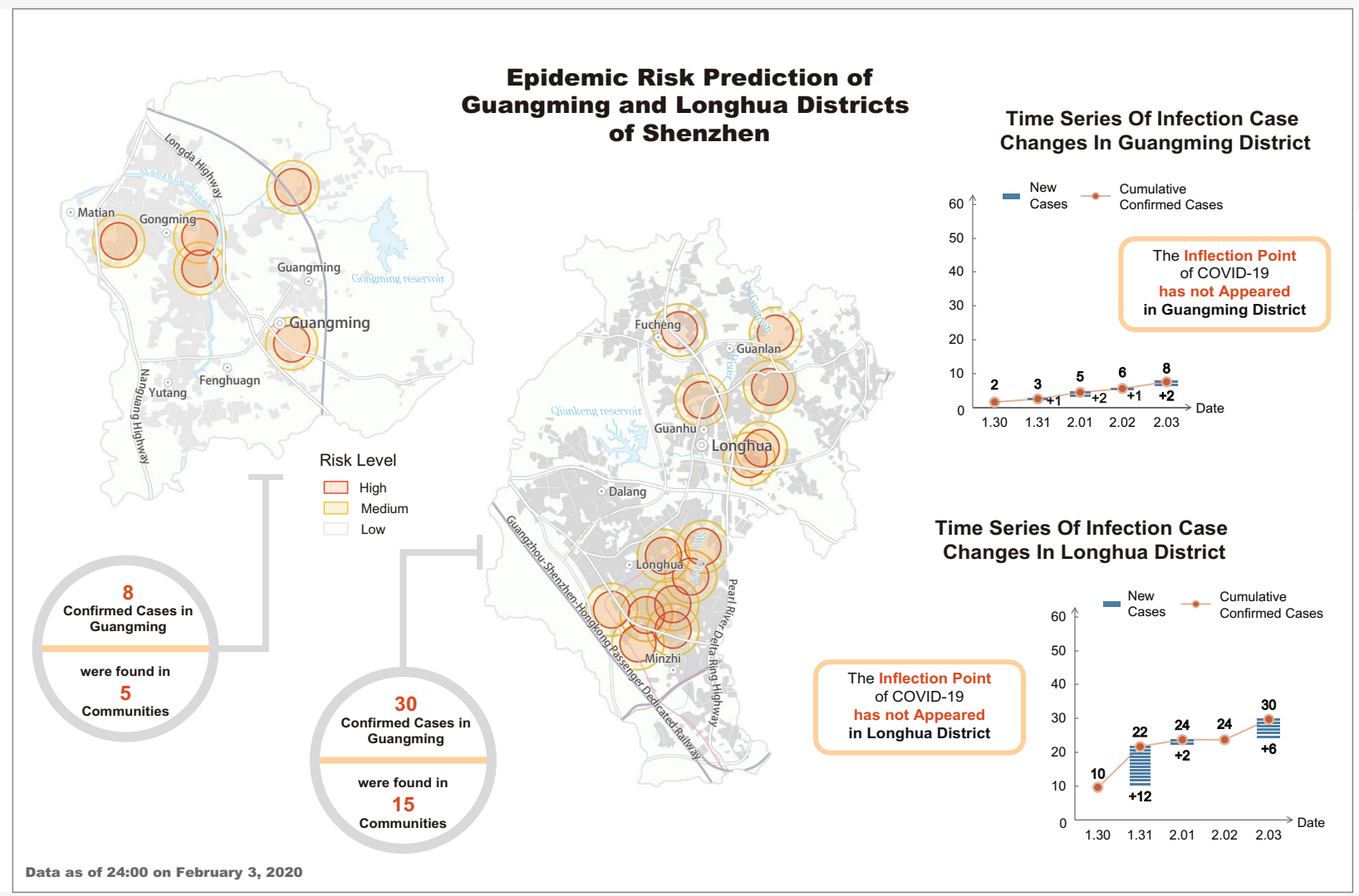

Fig. 11 Epidemic risk prediction of Guangming and Longhua districts of Shenzhen 
Funding This work was supported by the Fundamental Research Funds for the Central Universities (2042021kf0204).

Data Availability The epidemic data used and analyzed during the current study are available on the website of Shenzhen, China Government Data Open Platform, including the dataset on https://opendata.sz.gov. $\mathrm{cn} /$ data/api/toApi, and the online map on https://opendata.sz.gov.cn/ data/dataMap/toMap. The vector data in the geographical base map is available on the website of Shenzhen Platform for common Geospatial Information Services, including the standard maps on https://guang dong.tianditu.gov.cn/shenzhen/bzmap. All of the data used in this paper are either published online by relevant government departments and medical institutions or captured from the online map available through the open API interface.

Code Availability Not applicable.

\section{Declarations}

Ethical Approval Not applicable.

Informed Consent Not applicable.

Competing Interests The authors declare no competing interests.

\section{References}

Adams S, Morioka N, Stone TL (2008) Color design workbook: a realworld guide to using color in graphic design. Rockport Publishers, Gloucester, Mass

Akella MK (2009) First responders and crisis map symbols: clarifying communication. Cartogr Geogr Inf Sc 36(1):19-28. https://doi. org/10.1559/152304009787340179

Arco E, Ajmar A, Arneodo F, Boccardo P (2017) An operational framework to integrate traffic message channel (TMC) in emergency mapping services (EMS). Eur J Remote Sens 50(1):478495. https://doi.org/10.1080/22797254.2017.1361306

Ario AR, Makumbi I, Bulage L, Kyazze S, Kayiwa J, Wetaka MM, Ocom F (2019) The logic model for Uganda's health sector preparedness for public health threats and emergencies. Glob Health Action 12(1):7. https://doi.org/10.1080/16549716.2019.1664103

Brice J (2020) Charting COVID-19 futures: mapping, anticipation, and navigation. Dialogues Hum Geogr 10(2):271-275. https://doi.org/ $10.1177 / 2043820620934331$

Campbell-Lendrum D, Manga L, Bagayoko M, Sommerfeld J (2015) Climate change and vector-borne diseases: what are the implications for public health research and policy? Philos T R Soc B 370(1665):8. https://doi.org/10.1098/rstb.2013.0552

Chapin C, Sen Roy S (2021) A spatial web application to explore the interactions between human mobility, government policies, and COVID-19 cases. J Geovis Spat Anal 5(1):12. https://doi.org/10. 1007/s41651-021-00081-y

Elia A, Balbo S, Boccardo P (2018) A quality comparison between professional and crowdsourced data in emergency mapping for potential cooperation of the services. Eur J Remote Sens 51(1):572-586. https://doi.org/10.1080/22797254.2018.1460567

Fang H, Xin SW, Pang HS, Xu F, Gui YH, Sun Y, Yang N (2021) Evaluating the effectiveness and efficiency of risk communication for maps depicting the hazard of COVID-19. T Gis 00:1-24. https://doi.org/10.1111/tgis.12814
Girres JF (2019) Hazard-based images comparison methods for damage assessment in emergency mapping. Int J Cartogr 5(2-3):1-17. https://doi.org/10.1080/23729333.2019.1613075

Griffin AL, White T, Fish C, Tomio B, Huang H, Sluter CR, Bravo JVM, Fabrikant SI, Bleisch S, Yamada M (2017) Designing across map use contexts: a research agenda. Int J Cartogr 3(sup1):90114. https://doi.org/10.1080/23729333.2017.1315988

Guillette D, Stratton J, Varia M, Chau V, Loh LC (2020) Canadian public health agency lessons on using choropleth maps to characterize geographic distribution of COVID-19 data. Acta Medica Port 33(12):792-794. https://doi.org/10.20344/amp.15056

Katzburg JR, Fickel J, Bradley S, Lind J, Ong MK, Bergman A, Fleming M, Johnson E, Tubbesing SA (2020) Geographic information systems (GIS) mapping for emergency management in VHA home based primary care. J Am Geriatr Soc 68(SI):S51-S51

Kerkvoorde MV, Kellens W, Verfaillie E, Ooms K (2018) Evaluation of web maps for the communication of flood risks to the public in Europe. Int J Cartogr 4(1):49-64. https://doi.org/10.1080/23729 333.2017.1371411

Kim K, Jung K (2018) Dynamics of interorganizational public health emergency management networks: following the 2015 MERS response in South Korea. Asia-Pac J Public He 30(3):207-216. https://doi.org/10.1177/1010539518762847

Kostelnick JC, Hoeniges LC (2019) Map symbols for crisis mapping: challenges and prospects. Cartogr J 56(1):59-72. https://doi.org/ 10.1080/00087041.2017.1413810

Landa R (1996) Graphic design solutions. Cengage Learning, Boston

Li R (2021) Visualizing COVID-19 information for public: designs, effectiveness, and preference of thematic maps. Human Behav Emerg Technol 3(1):97-106. https://doi.org/10.1002/hbe2.248

Liu F, Liu D (2020) Visualization Mapping Analysis of Emergency Management Scientific Research Big Data during COVID19. Paper presented at the 5th International Conference on Mechanical, Control and Computer Engineering (ICMCCE), Harbin, Peoples R China. https://doi.org/10.1109/icmcce51767. 2020.00506

Lovett DA, Poots AJ, Clements J, Green SA, Samarasundera E, Bell D (2014) Using geographical information systems and cartograms as a health service quality improvement tool. Spat Spatiotemporal Epidemiol 10(1):67-74. https://doi.org/10.1016/j.sste.2014. 05.004

Malpass M (2016) Critical design in context: history, theory, and practices. Bloomsbury, London

Marome W, Shaw R (2021) COVID-19 response in Thailand and its implications on future preparedness. Int J Environ Res Pu 18(3):1089. https://doi.org/10.3390/ijerph18031089

Melin P, Monica J C, Sanchez D, Castillo O (2020) Analysis of spatial spread relationships of coronavirus (COVID-19) pandemic in the world using Self Organizing Maps. Chaos Soliton Fract 138https://doi.org/10.1016/j.chaos.2020.109917

Pelizza A (2020) "No disease for the others": how COVID-19 data can enact new and old alterities. Big Data Soc 7(2):7. https://doi.org/ $10.1177 / 2053951720942542$

Rao J, Chen K, Yang EF, Kruse J, Hudson K, Gao S (2021) A multiperspective narrative-based geovisualization dashboard for the 2020 US presidential election. J Geovis Spat Anal 5(2):15. https:// doi.org/10.1007/s41651-021-00087-6

Talisuna AO, Okiro EA, Yahaya AA, Stephen M, Bonkoungou B, Musa EO, Minkoulou EM, Okeibunor J, Impouma B, Djingarey HM, Yao NKM, Oka S, Yoti Z, Fall IS (2020) Spatial and temporal distribution of infectious disease epidemics, disasters and other potential public health emergencies in the World Health Organisation Africa region, 2016-2018. Globalization Health 16(1):9. https://doi.org/10.1186/s12992-019-0540-4

Vassileva M, Tonolo FG, Riccardi P, Lecci D, Boccardo P, Chiesa G (2017) Satellite SAR interferometric techniques in support to 
emergency mapping. Eur J Remote Sens 50(1):464-477. https:// doi.org/10.1080/22797254.2017.1360155

Weng M, Song X, Wang L, Xie H, Zhang P, Su S, Kang M (2020) A tourist map of Xi' an: combining historical city characteristics with art. J Maps 16(1):195-202. https://doi.org/10.1080/17445 647.2020.1837269

Wildbur P, Burke M (1998) Information graphics: innovative solutions in contemporary design. Thames and Hudson, London

Xiao Y (2020) Challenges and response proposals on public health emergency legal system under COVID-19 epidemic. Bull Chinese Acad Sci 35(3):240-247. https://doi.org/10.16418/j.issn. 1000-3045.20200218001

Xu C, Zhang X, Wang Y (2020a) Mapping of health literacy and social panic via web search data during the COVID-19 public health emergency: Infodemiological study. J Med Internet Res 22(7):e18831. https://doi.org/10.2196/18831

Xu J, Zhou H, Nie G, An J (2020b) Plotting earthquake emergency maps based on audience theory. Int J Disast Risk Re 47:101554. https://doi.org/10.1016/j.ijdrr.2020.101554

Yalcin M (2020) Mapping the global spatio-temporal dynamics of COVID-19 outbreak using cartograms during the first 150 days of the pandemic. Geocatro Int 1:1-10. https://doi.org/10.1080/ 10106049.2020.1844310

Yang JY, Shi BX, Xia GY, Xue Q, Yang XF (2021) Construction of a double-scale epidemic prevention system with consideration of city and community. Chin Sci Bull 66(4-5):433-438. https://doi. org/10.1360/tb-2020-1071

Ye X, Du J, Gong X, Na S, Li W, Kudva S (2021) Geospatial and semantic mapping platform for massive COVID-19 scientific publication search. J Geovis Spat Anal 5(1):12. https://doi.org/ 10.1007/s41651-021-00073-y

Zhang L, Li H, Chen K (2020) Effective risk communication for public health emergency: reflection on the COVID-19 (2019-nCoV) outbreak in Wuhan, China. Healthcare 8(1) https://doi.org/10.3390/ healthcare8010064

Zhou M, Hu W, Ai T (2020) Multi-level thematic map visualization using the Treemap hierarchical representation model. J Geovis Spat Anal 4(1):11. https://doi.org/10.1007/s41651-020-00053-8

Publisher's Note Springer Nature remains neutral with regard to jurisdictional claims in published maps and institutional affiliations. 